\title{
Nonrepetitively 3-colorable subdivisions of graphs with a logarithmic number of subdivisions per edge
}

\author{
Matthieu Rosenfeld \\ LIRMM, CNRS, Université de Montpellier \\ matthieu.rosenfeld@gmail.com
}

Submitted: Apr 16, 2021; Accepted: Oct 21, 2021; Published: Nov 5, 2021

(C) The author. Released under the CC BY-ND license (International 4.0).

\begin{abstract}
We show that for every graph $G$ and every graph $H$ obtained by subdividing each edge of $G$ at least $\Omega(\log |V(G)|)$ times, $H$ is nonrepetitively 3-colorable. In fact, we show that $\Omega\left(\log \pi^{\prime}(G)\right)$ subdivisions per edge are enough, where $\pi^{\prime}(G)$ is the nonrepetitive chromatic index of $G$. This answers a question of Wood and improves a similar result of Pezarski and Zmarz that stated the existence of at least one 3-colorable subdivision with a linear number of subdivision vertices per edge.
\end{abstract}

Mathematics Subject Classifications: 05C15

\section{Introduction}

A sequence $s_{1} \ldots s_{2 n}$ is a square if $s_{i}=s_{i+n}$ for each $i \in\{1, \ldots, n\}$. A sequence is repetitive if it contains a consecutive subsequence that is a square and it is nonrepetitive (or square-free) otherwise. For instance, the words hotshots, repetitive and alfalfa are repetitive and the words total and minimize are nonrepetitive.

The work of Thue on nonrepetitive words is regarded as the starting point of combinatorics on words [15, 16] (see [4] for a translation in modern mathematical English). He showed that there are infinite square-free sequences over three elements. Many generalizations and variations of this notion have been studied. In particular, the notion of nonrepetitive coloring of graphs was introduced by Alon et al. [1] (see [17] for a recent survey on this topic). We say that a coloring (either of the vertices or of the edges) of a graph is nonrepetitive if the sequence of colors of any path is nonrepetitive. The nonrepetitive chromatic number (resp. nonrepetitive chromatic index) of a graph, denoted by $\pi(G)$ (resp. $\pi^{\prime}(G)$ ) is the smallest number of colors in a nonrepetitive coloring of the vertices (resp. the edges) of the graph. Alon et al. showed that $\pi^{\prime}(G)$ is in $O\left(\Delta^{2}\right)$ where $\Delta$ is the maximum degree of $G$ [1]. Different authors successively improved the upper bounds on the nonrepetitive chromatic number and the nonrepetitive chromatic index and the best known bound for the nonrepetitive chromatic number is also in $O\left(\Delta^{2}\right)[5,7,9,13]$. 
Nonrepetitive colorings of subdivisions of graphs were also widely studied. We say that a graph $G^{\prime}$ is a subdivision of the graph $G$ if $G^{\prime}$ is obtained by replacing each edge $v w$ of $G$ by a path $P$ with endpoints $v$ and $w$, where the new paths are pairwise internally disjoints. If each edge is replaced by a path with at least $d$ internal vertices then $G^{\prime}$ is a $(\geqslant d)$-subdivision of $G$. Barát and Wood proved that every graph has a nonrepetitively 4-colorable subdivision [2]. Pezarski and Zmarz reduced 4 to 3 [12] (solving a conjecture of Grytczuk [8]). This result is a strong generalization of Thue's result. In these two results, the number of division vertices per edge is $O(|V(G)|)$ or $O(|E(G)|)$. Djumović et al. showed that every graph has a nonrepetitively 5-colorable subdivision with $O(\log |V(G)|)$ division vertices per edge [5]. Their result is in fact stronger than that since it holds in the list-coloring setting, and they only require that each edge $v w$ is subdivided at least $c \log (\operatorname{deg}(v) \operatorname{deg}(w))$ times for some constant $c$. In particular, it applies to every subdivision with at least a certain number of division vertices per edge which was not the case for the previous results. Finally, Wood proved that every graph has a nonrepetitively 5-colorable subdivision with $O(\log \pi(G))$ division vertices per edge [17]. It is slightly stronger since it implies the same bound of $O(\log \Delta)$, but it does not hold in the listcoloring setting and requires all the edges to be subdivided the same number of times. In a recent survey Wood asked the following question.

Question 1. [17, Open Question 6.21] Does every graph $G$ have a nonrepetitively 3colourable subdivision with $O(\log |V(G)|))$ or even $O(\log \pi(G))$ division vertices per edge?

In this article, we give a positive answer to the first part of this question. In fact, we show that there exists a function $f(n)=O(\log n)$ such that any subdivision of any graph $G$ with at least $f\left(\pi^{\prime}(G)\right)$ subdivision per edge is nonrepetitively 3-colorable. Since $\pi^{\prime}(G)$ is in $O\left(\Delta^{2}\right)$, the quantity $\left.O\left(\log \pi^{\prime}(G)\right)\right)$ is smaller than $\left.O(\log \Delta)\right)$ which is itself smaller than the suggested $O(\log |V(G)|))$. However, $\log \pi^{\prime}(G)$ can be arbitrarily larger than $\log \pi(G)$ (let $K_{1, t}$ be the star over $k+1$ vertices, then $\pi\left(K_{1, t}\right)=2$ and $\pi^{\prime}\left(K_{1, t}\right)=t$ ), so we are not able to solve the second part of this question. The number of subdivision vertices per edge that we require is $558 \log _{2}(n)+O(1)$. This result is optimal in the sense that $\Omega(\log n)$ division vertices are needed on some edges of any nonrepetitively $O(1)$-colorable subdivision of $K_{n}[10]$. However, we expect the optimal multiplicative coefficient in front of the log to be much smaller than 558 .

Moreover, we show that any subdivision is nonrepetitively colorable as long as each edge is subdivided at least a certain number of times. This is much stronger than showing that there exists one nonrepetitively colorable subdivision. The results of [2] and [12] only imply the existence of one nonrepetitively colorable subdivision with the desired property. Our proof becomes much simpler if we only care about the existence of one nonrepetitively 3-colorable subdivision.

The article is organized as follows. We first provide in Section 2 some definitions and recall some useful results from the literature. Then in Section 3, we show the existence of a set of words that is needed for our main construction. In particular, we show that there are sets of $n$-good words of exponential size. In Section 4 , we use these sets of $n$-good words to show our main result. The main idea is to start from a nonrepetitive coloring 
of the edges of a graph and to "encode" each color by a well-chosen $n$-good word. In the last section, we discuss possible ways to improve the bound on the number of needed subdivisions per edge.

The main idea is implicitly to generalize the notion of square-free morphism. A morphism is a map $h: \Sigma^{*} \rightarrow \Sigma^{*}$ such that the image of each word is given by the concatenation of the image of the letters. A square-free morphism is a morphism such that the image of every square-free word is a square-free word. A directed graph is nonrepetitively colored if the sequence of colors of every directed path is nonrepetitive. Given a directed graph $D$, two sets of colors $C$ and $\Sigma$, a nonrepetitive edge coloring $\phi: A(D) \rightarrow C$ of $D$ and a square-free morphism $h: C \mapsto \Sigma$. If we can subdivide $D$ in such a way that the sequence of colors of the subdivision of any edge $e$ is $h(\phi(e))$, then this subdivision is nonrepetitively $|\Sigma|$-colorable ${ }^{1}$. We cannot use the same construction for undirected graphs because the subdivision of an edge can appear in "the wrong direction" in a path. But if we can find a square-free morphism such that the image of every square-free word is square-free even if we replace the image of a letter with its mirror at any set of arbitrary positions, then we can use the same idea. There are extra technicalities, since we show that it works for any large enough subdivision. Naturally, the proof mostly relies on combinatorics on words.

\section{Preliminaries}

A word is a finite sequence over a finite set that we call the alphabet. A factor of a word is a contiguous subsequence of this word, that is, if there are two words $p$ and $s$ such that $w=p f s$ then $f$ is a factor of $w$. If $p$ (resp. $s$ ) is empty then $f$ is also a prefix (resp. $a$ suffix) of $w$. A prefix (resp. a suffix) of $w$ is proper if it is not equal to $w$. The length of a word $u$ is denoted by $|u|$. A word $u$ occurs in $v$ at position $p$, if the factor of length $|u|$ of $v$ that starts at position $p$ is exactly $u$. The mirror image $\bar{w}$ of a word $w$ is the word obtained by reading $w$ from right to left. We let $\bar{w}^{1}=\bar{w}$ and $\bar{w}^{0}=w$.

We recall the following result from Shur on the number of square-free ternary words.

Theorem 2 ([14]). For all $n$, let $C_{s q}(n)$ be the number of ternary square-free words of length $n$. Then

$$
\limsup _{n \rightarrow \infty} C_{s q}(n)^{\frac{1}{n}} \geqslant 1,30175907 .
$$

Since any factor of a square-free word is square-free, $C_{s q}$ is a submultiplicative function (i.e., we have $C_{s q}(i+j) \leqslant C_{s q}(i) C_{s q}(j)$, for all $\left.i, j\right)$. By Fekete's Lemma, we deduce the following Corollary of Theorem 2.

Corollary 3. For all integer $n \geqslant 1, C_{s q}(n)>1.3^{n}$.

We now recall Turán's Theorem and a simple corollary.

\footnotetext{
${ }^{1}$ We might need one extra color to color the vertices from the original graph.
} 
Theorem 4 (Turán's Theorem). Let $G$ be any graph with $n$ vertices, such that $G$ is $K_{r+1}$-free. Then the number of edges in $G$ is at most

$$
|E(G)| \leqslant\left(1-\frac{1}{r}\right) \frac{n^{2}}{2}
$$

Corollary 5. Any graph $G$ contains an independent set of size at least $\left\lceil\frac{n}{1+d(G)}\right\rceil$ where $d(G)$ is the average degree of $G$.

Proof. Let $G$ be a graph of average degree $d(G)$ and let $H$ be the complement of $G$. Then the number of edges of $H$ is

$$
\begin{aligned}
|E(H)|=\frac{n(n-1)}{2}-|E(G)|=\frac{n(n-1)}{2}-\frac{n d(G)}{2} & =\frac{n^{2}}{2}\left(1-\frac{1+d(G)}{n}\right) \\
& >\frac{n^{2}}{2}\left(1-\frac{1}{\left\lceil\frac{n}{1+d(G)}\right\rceil-1}\right) .
\end{aligned}
$$

By Turán's Theorem, this implies that $H$ cannot be $K_{\left\lceil\frac{n}{1+d(G)}\right.}$-free. Hence, $G$ contains an independent set of size $\left\lceil\frac{n}{1+d(G)}\right\rceil$ as desired.

\section{Good sets}

Let $\sigma=1202120121021201021, \bar{\sigma}=1201021201210212021$ be the mirror image and let $\rho=\bar{\sigma} 0 \sigma$. By construction $\rho$ is a palindrome, $|\sigma|=19$ and $|\rho|=39$.

A word $v$ is nice if $\rho v \rho$ is square-free and contains only two occurrences of $\sigma$ and two occurrences of $\bar{\sigma}$. The only occurrences of $\sigma$ and $\bar{\sigma}$ are inside the two occurrences of $\rho$. Let $\mathcal{N}$ be the set of nice words and for any integer $n$, let $\mathcal{N}_{n}$ be the set of nice words of length $n$.

For all $n \geqslant 7200$, let $l_{n}$ be the lexicographically least word of the set $\mathcal{N}_{n}^{2}$. We show in Lemma 14 that there exist nice words of every length at least 7200 . So $l_{n}$ is properly defined for any $n \geqslant 7200$.

A set of words $S$ is mirror-free if for any word $w$ from $S$ such that $w \neq \bar{w}, \bar{w}$ is not in $S$. For any integer $n \geqslant 7200$, a set of words $\mathcal{S} \subseteq \mathcal{N}_{n}$ is $n$-good if it is mirror-free and if for all $i \in\{2 n+100, \ldots, 7 n\}$ and all $u, v \in \mathcal{S}$ such that $u \neq v$ the words $\rho u \rho v \rho l_{i}, \rho \bar{u} \rho v \rho l_{i}$, $\rho u \rho \bar{v} \rho \bar{l}_{i}$ and $\rho \bar{u} \rho \bar{v} \rho \bar{l}_{i}$ are square-free.

In Lemma 7, we show the central property of $n$-good set, but we first show the following Lemma as a warm-up exercise.

Lemma 6. Let $n \geqslant 7200$ be an integer, $\mathcal{S}$ be an $n$-good set, $u, v \in \mathcal{S}$ and $i \geqslant 2 n+100$ be an integer then $\rho u \rho l_{i} \rho v \rho$ is square-free.

\footnotetext{
${ }^{2}$ We only need to make an arbitrary choice to pick one element of each $\mathcal{N}_{n}$, but we never use the fact that $l_{n}$ is the lexicographically least word of $\mathcal{N}_{n}$.
} 
Proof. Suppose, for the sake of contradiction, that $\rho u \rho l_{i} \rho v \rho$ contains a square $w w$. First remark, that since $\mathcal{S}$ is an $n$-good set $\rho u \rho l_{i} \rho$ and $\rho l_{i} \rho v \rho$ are both square-free. Hence there exist a non-empty suffix $x$ of $\rho u$ and a non-empty prefix $y$ of $v \rho$ such that $x \rho l_{i} \rho y$ is a square.

Now $l_{i} \rho$ is not a factor of $w$ since it contains only one occurrence of $\rho$ and that $l_{i}$ is much longer than the gap between any other occurrences of $\rho$. For the same reason, $\rho l_{i}$ is not a factor of $w$. There exists $x^{\prime}, y^{\prime}$ such that $l_{i}=x^{\prime} y^{\prime}$ and $w=x \rho x^{\prime}=y^{\prime} \rho y$. Since $\left|x^{\prime}\right|+\left|y^{\prime}\right|=\left|l_{i}\right| \geqslant 2 n+100$, assume, without loss of generality that $\left|x^{\prime}\right| \geqslant n+50$. Then $\left|x^{\prime}\right|>|y|$ and $x p$ is a proper prefix of $y^{\prime} \rho$ which implies that there is a second occurrence of $\rho$ in $y^{\prime} \rho$. This contradicts the fact that $l_{i}$ is a nice word.

Lemma 7. Let $k$ and $n$ be two integers. Let $\mathcal{S}$ be an $n$-good set, $\Sigma$ be an alphabet and $f: \Sigma \mapsto \mathcal{S}$ be an injective map. Then, for any square-free word $w_{1} \ldots w_{k} \in \Sigma^{k}$, any sequence of integers $\left(s_{i}\right)_{1 \leqslant i \leqslant k} \in\{2 n+100, \ldots, 7 n\}^{k}$ and any sequence $\left(r_{i}\right)_{1 \leqslant i \leqslant k} \in\{0,1\}^{k}$ the word

$$
\rho \prod_{i=1}^{k}{\overline{f\left(w_{i}\right) \rho l_{s_{i}} \rho f\left(w_{i}\right)}}^{r_{i}} \rho=\rho{\overline{f\left(w_{1}\right) \rho l_{s_{1}} \rho f\left(w_{1}\right)}}^{r_{1}} \rho \ldots{\overline{f\left(w_{k}\right) \rho l_{s_{k}} \rho f\left(w_{k}\right)}}^{r_{k}} \rho
$$

is square-free.

Proof. Suppose, for the sake of contradiction, that $\rho \prod_{i=1}^{k}{\overline{f\left(w_{i}\right) \rho l_{t_{i}} \rho f\left(w_{i}\right)}}^{r} \rho$ contains a square $u u$. There are $x_{1}, \ldots, x_{3 k} \in \mathcal{N}$ such that for all $i$,

$$
{\overline{f\left(w_{i}\right) \rho l_{s_{i}} \rho f\left(w_{i}\right)^{r}}}^{r_{i}}=x_{3 i-2} \rho x_{3 i-1} p x_{3 i} .
$$

It implies

$$
\rho \prod_{i=1}^{k}{\overline{f\left(w_{i}\right) \rho l_{s_{i}} \rho f\left(w_{i}\right)}}^{r_{i}} \rho=\rho x_{1} \rho x_{2} \rho \ldots \rho x_{3 k} \rho
$$

and there are no other occurrences of $\sigma$ and $\bar{\sigma}$ than the $3 k+1$ occurrences that are inside the occurrences of $\rho$.

Let $L=\left\{l_{i}: i \in\{2 n+100, \ldots, 7 n\}\right\}$. Then for any $j, x_{j} \in L$ if and only if $j \equiv 2$ $\bmod 3$. Since $\mathcal{S}$ is mirror-free, for any $i, x_{i}$ and $x_{i+1}$ are different. Thus, by the definition of $n$-good sets and by Lemma 6 , for any $i, \rho x_{i} \rho x_{i+1} \rho x_{i+2} \rho$ is square-free. Thus there are at least 3 occurrences of $\rho$ in $u$. For the rest of the proof assume that the middle of the square $u u$ does not cut any occurrence of $\sigma$. If this is not true, it implies that the middle of the square $u u$ does not cut any occurrence of $\bar{\sigma}$, and the proof is identical (consider the mirror of the whole world in which the middle of the square $\overline{u u}$ does not cut any occurrence of $\sigma$ ). Then $\sigma$ occurs at least twice in $u$.

Let $l \geqslant 2$ be the number of occurrences of $\sigma$ in $u$. The occurrences of $\sigma$ in the two consecutive occurrences of $u$ need to be matched, so in particular there are as many occurrences of $\sigma$ in the two occurrences of $u$. Hence, there exist an integer $m \in\{l, \ldots, 3 k+$ $1-l\}$ such that

$$
\begin{aligned}
u & =y \sigma x_{m+1-l} \bar{\sigma} 0 \sigma x_{m+2-l} \bar{\sigma} 0 \sigma \ldots \bar{\sigma} 0 \sigma x_{m-1} \bar{\sigma} 0 \sigma y^{\prime} \\
& =z \sigma x_{m+1} \bar{\sigma} 0 \sigma x_{m+2} \bar{\sigma} 0 \sigma \ldots \bar{\sigma} 0 \sigma x_{m+l-1} \bar{\sigma} 0 \sigma z^{\prime}
\end{aligned}
$$


where $y$ is a proper suffix of $\sigma x_{m-l} \bar{\sigma} 0$ (or a suffix of $\bar{\sigma} 0$, if $m-l=0$ ), $y^{\prime} z=x_{m} \bar{\sigma} 0$ and $z^{\prime}$ is a prefix of $x_{m+l} \bar{\sigma} 0$ (or a prefix of $\varepsilon$, if $m-l=3 k+1$ ). For any $j$, the factors between the $j$ th and the $(j+1)$ th occurences of $\sigma$ in the two occurences of $u$ are equal, that is, for all $j \in\{0, \ldots, l-2\}$,

$$
x_{m+1-l+j}=x_{m+1+j}
$$

and

$$
y=z \text { and } y^{\prime}=z^{\prime} .
$$

Recall that, for any $j, x_{j} \in L$ if and only if $j \equiv 2 \bmod 3$.

Let us first deal with the case $l=2$. In this case,

$$
u=y \sigma x_{m-1} \bar{\sigma} 0 \sigma y^{\prime}=z \sigma x_{m+1} \bar{\sigma} 0 \sigma z^{\prime} .
$$

Then (2) implies $x_{m-1}=x_{m+1}$ which is only possible if $m \equiv 2 \bmod 3$ (otherwise exactly one of $x_{m-1}$ and $x_{m+1}$ is in $L$ which contradicts the equality). Since $m-2 \equiv 0 \bmod 3$, $|y|<n+39$. In deed, if $m-2 \geqslant 1$, then $y$ is a proper suffix of $\sigma x_{m-2} \bar{\sigma} 0$ and $|y|<$ $\left|\sigma x_{m-2} \bar{\sigma} 0\right|=n+39$, and if $m-2=0$, then $y$ is a suffix of $\bar{\sigma} 0$ and $|y| \leqslant 20$. A similar argument based on $m+2 \equiv 1 \bmod 3$ leads to $\left|z^{\prime}\right| \leqslant n+20$. Since $x_{m} \bar{\sigma} 0=y^{\prime} z$, equation (2) implies

$$
\left|x_{m}\right|=|y|+\left|z^{\prime}\right|-|\bar{\sigma} 0|<2 n+59 .
$$

But $m \equiv 2 \bmod 3$ implies $\left|x_{m}\right| \geqslant 2 n+100$ which contradicts the previous equation.

Let us now take care of the case $l \geqslant 3$. We first show that $l \equiv 0 \bmod 3$. Recall, that for all $j,\left|x_{j}\right| \neq n$ if and only if $j \equiv 2 \bmod 3$. Together with (1), it implies that for all $j \in\{0, \ldots, l-2\}, m+1-l+j \equiv 2 \bmod 3$ if and only if $m+1+j \equiv 2 \bmod 3$. Thus if $m+1-l \equiv 2 \bmod 3$ then $m+1 \equiv 2 \bmod 3$ and $l \equiv 0 \bmod 3$. If $m+1-l \equiv 1 \bmod 3$, then $m+2-l \equiv 2 \bmod 3$ and $m+2 \equiv 2 \bmod 3$ which also implies $l \equiv 0 \bmod 3$. If $m+1-l \equiv 0 \bmod 3,\left|x_{m+1-l}\right|=\left|x_{m+2-l}\right|=n$ which implies $\left|x_{m+1}\right|=\left|x_{m+2}\right|=n$ and $m+1 \equiv 0 \bmod 3$ and finally $l \equiv 0 \bmod 3$.

We have three distinct cases to consider.

Case $\boldsymbol{m}+\mathbf{1}-\boldsymbol{l} \equiv \mathbf{1} \bmod 3: \quad$ For every $j \equiv 1 \bmod 3, x_{j}={\overline{f\left(w_{(j+2) / 3}\right)^{(j+2) / 3}}}^{r}$. Thus for all integer $j, x_{m+1+3 j-l}={\overline{f\left(w_{(m-l) / 3+1+j}\right)^{r(m-l) / 3+1+j}}}^{r}$ and $x_{m+1+3 j}={\overline{f\left(w_{m / 3+1+j}\right)}}^{r_{m / 3+1+j}}$. By equation (1), for all $j \in\{0, \ldots, l / 3-1\}$,

$$
{\overline{f\left(w_{(m-l) / 3+1+j}\right)}}^{r(m-l) / 3+1+j}={\overline{f\left(w_{m / 3+1+j}\right)}}^{r / 3+1+j} .
$$

The function $f$ is an injective map to $\mathcal{S}$ which is mirror-free, so the previous equation implies that for all $j \in\{0, \ldots, l / 3-1\}$,

$$
w_{(m-l) / 3+1+j}=w_{m / 3+1+j} .
$$

This implies that there is a square in $w$ which is a contradiction. 
Case $\boldsymbol{m}+\mathbf{1}-\boldsymbol{l} \equiv \mathbf{0} \bmod 3$ : We use the same idea as in the previous case with the fact that for every $j \equiv 0 \bmod 3, x_{j}={\overline{f\left(w_{j / 3}\right)}}^{r_{j / 3}}$. In this case we obtain that for all $j \in\{0, \ldots, l / 3-1\}$,

$$
w_{(m+1-l) / 3+j}=w_{(m+1) / 3+j} .
$$

This implies that there is a square in $w$ which is a contradiction.

Case $\boldsymbol{m}+\mathbf{1}-\boldsymbol{l} \equiv \mathbf{2} \bmod 3$ : This case is almost identical to the previous ones. We know that for every $j \equiv 0 \bmod 3, x_{j}={\overline{f\left(w_{j / 3}\right)}}^{r_{j / 3}}$. Moreover for all $j, m+2-l+3 t \equiv 0$ mod 3. By the same argument, for all $j \in\{0, \ldots, l / 3-1\}$,

$$
w_{(m+2-l) / 3+j}=w_{(m+2) / 3+j} .
$$

This implies that there is a square in $w$ which is a contradiction.

This property is essential to construct the nonrepetitive coloring of a subdivided graph. The idea is to encode the colors of the edges of a nonrepetitive edge coloring of the initial graph. Any vertex from the initial graph will be colored by 0 and the path corresponding to any edge colored $c$ in the original graph should receive the color sequence $\sigma f(c) \rho l_{i} \rho f(c) \bar{\sigma}$ (with the $l_{i}$ of the right length). The fact that we can choose a different $l_{i}$ for every edge means that we can find a valid encoding as long as the edge is subdivided enough. The fact that we can replace the encoding of each $w_{i}$ by the mirror image of the encoding means that we can take an arbitrary orientation of the subdivided edge to apply the encoding to the corresponding path.

We also need a variant of this property. This variant will be useful for paths that start and end in the subdivision of the same edge (i.e., paths that appear in the subdivision of a cycle).

Lemma 8. Let $k \geqslant 3$ and $n$ be two integers. Let $S$ be an $n$-good set, $\Sigma$ be an alphabet and $f: \Sigma \mapsto S$ be an injective map. Let $w_{1} \ldots w_{k} \in \Sigma^{k}$ be a square-free word such that $w_{2} w_{3} \ldots w_{k} w_{1}$ is also square-free. Let $\left(t_{i}\right)_{1 \leqslant i \leqslant k} \in\{2 n+100, \ldots, 7 n\}^{k}$ be a sequence of integers, and $\left(r_{i}\right)_{1 \leqslant i \leqslant k} \in\{0,1\}^{k}$ be a sequence of 0 and 1 . Let $a$ and $b$ be a pair of words such that $\overline{f\left(w_{1}\right) \rho l_{t_{1}} \rho f\left(w_{1}\right)}{ }^{r}=a b$. Then the word

$$
\begin{aligned}
& b \rho\left(\prod_{i=2}^{k}{\overline{f\left(w_{i}\right) \rho l_{t_{i}} \rho f\left(w_{i}\right)}}^{r_{i}} \rho\right) a \\
& =b \rho \overline{f\left(w_{2}\right) \rho l_{t_{2}} \rho f\left(w_{2}\right)}{ }^{r_{2}} \rho{\overline{f\left(w_{2}\right) \rho l_{t_{2}} \rho f\left(w_{2}\right)}}^{r_{2}} \rho \ldots \rho \overline{f\left(w_{k}\right) \rho l_{t_{k}} \rho f\left(w_{k}\right)}{ }^{r_{k}} \rho a
\end{aligned}
$$

is square-free.

Proof. Suppose, for the sake of contradiction, that $b \rho\left(\prod_{i=2}^{k}{\overline{f\left(w_{i}\right) \rho l_{t_{i}} \rho f\left(w_{i}\right)}}^{r_{i}} \rho\right) a$ contains a square $u$.

Since $w_{1} w_{2} \ldots w_{k}$ and $w_{2} w_{3} \ldots w_{k} w_{1}$ are both square-free, Lemma 7 implies that

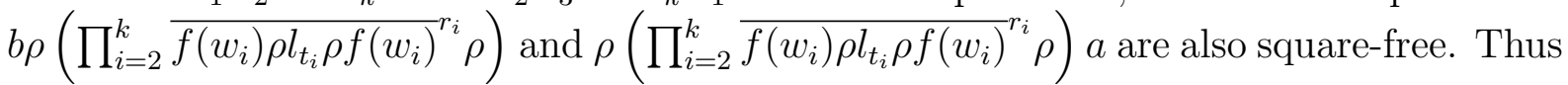
the first occurrence of $u$ starts in $b$ and the second occurrence ends in $a$. Assume, without 
loss of generality, that the middle of the square does not cut an occurrence of $\sigma$. Since $k \geqslant 3$, we know that $u u$ contains at least 8 occurrences of $\sigma$ and by the same argument as in the preivous proof it implies that the number of occurences of $\sigma$ in $u$ is a multiple of 3. The number of $\sigma$ is necessarily a multiple of 6 and it is also equal to $3 k-x$ where $x \in\{0,1,2\}$ is the number of $\sigma$ located in $a b$ and ommited by $u u$. It implies that $k$ is even and $x=0$ so $u u$ contains all the occurences of $\sigma$.

From here, with an argument similar to the proof of Lemma 7 one easily shows that for every $i \in\left\{1, \ldots, \frac{k}{2}-1\right\}$,

$$
w_{i+1}=w_{k / 2+i+1} .
$$

Then one can similarily verify that $\overline{f\left(w_{k / 2+1}\right) \rho l_{t_{k / 2+1}} \rho f\left(w_{k / 2+1}\right)}{ }^{r_{k / 2+1}}$ is necessarily a factor of $a b={\overline{f\left(w_{1}\right) \rho l_{t_{1}} \rho f\left(w_{1}\right)^{r}}}^{r_{1}}$. This is only possible if $w_{1}=w_{k / 2+1}$. Thus, for every $i \in$ $\left\{0, \ldots, \frac{k}{2}-1\right\}$,

$$
w_{i+1}=w_{k / 2+i+1} \text {. }
$$

This is a contradiction since $w_{1} \ldots w_{k}$ is square-free.

\subsection{Exponentially large good sets}

We show in this subsection that there are exponentially many nice words of any length and we use that to show that there are exponentially large $n$ good sets.

Let $h: \Sigma \mapsto \Sigma^{*}$, be the map such that

$$
\begin{aligned}
& h(0)=\{012102120210201021201210,0121021202102012021201210\}, \\
& h(1)=\{120210201021012102012021,1202102010210120102012021\} \\
& h(2)=\{201021012102120210120102,2010210121021201210120102\} .
\end{aligned}
$$

A word $v$ is an image of $w$ by $h$ if $v$ can be obtained by replacing each occurrence of any letter $i$ of $w$ by any word of the corresponding set $h(i)$. The set of images of $w$ by $h$ is denoted by $h(w)$. The authors of [3] introduced $h$ and showed the following property. ${ }^{3}$

Theorem 9 ([3, Theorem 20]). For any square-free word $w \in\{0,1,2\}^{*}$ and any $v \in h(w)$, $v$ is square-free.

We will use $h$ to show that the set of nice words has exponential growth. First, we need a few simple facts about $h, \sigma$ and $\rho$.

Lemma 10. The following facts holds.

1. For any letters $a, b, c \in\{0,1,2\}, v \in h(a b)$ and $v^{\prime} \in h(c)$, the word $v^{\prime}$ cannot occur as an internal factor of $v$ (i.e., the only possible occurrences of $v^{\prime}$ are as suffix or prefix).

2. For any $a, b \in\{0,1,2\}$ and $v \in h(a b)$, neither $\sigma$ or $\bar{\sigma}$ are factor of $v$.

\footnotetext{
${ }^{3}$ In fact, the map that they used contains 4 words in each set and here we used only 2 of them.
} 
3. If $a \in\{1,2\}, b \in\{0,1,2\} \backslash\{a\}$ and $v \in h(0 a b)$, then the word $\rho v$ is square-free and contains exactly one occurrence of $\sigma$ and $\bar{\sigma}$.

4. If $a \in\{1,2\}, b \in\{0,1,2\} \backslash\{a\}$ and $v \in h(b a 0)$, then the word $v \rho$ is square-free and contains exactly one occurrence of $\sigma$ and $\bar{\sigma}$.

It is a bit tedious to verify the four claims of this Lemma by hand so we provide a simple computer program that verifies Lemma $10^{4}$.

The first of these 4 facts implies that images by $h$ synchronize, that is, as long as a factor of an image is of length at least 50 (the length of the images of two letters), there is a unique way to split it into different images by $h$. These kinds of properties are really useful to establish that an image of a square-free word by $h$ does not contain any large squares (i.e., squares that are large enough to allow us to use this synchronization property). On the other hand, the other facts are quite useful to establish that there are no short squares. With this lemma in hand, it is relatively simple to provide a proof of Theorem 9 (which we will not do). We can use these facts to show that there are exponentially many nice words.

Lemma 11. Let $w \in\{0,1,2\}^{*}$ be a word such that $|w| \geqslant 4$ and $10 w 01$ is square-free. Then any $v \in h(0 w 0)$ is nice.

Proof. Let $w$ and $v$ be as in the Theorem statement. Let $w_{1}, \ldots, w_{n} \in\{0,1,2\}$ be such that $w_{1} \ldots w_{n}=0 w 0$ and for all $i$ let $v_{i} \in h\left(w_{i}\right)$ such that $v=v_{1} \ldots v_{n}$. Recall that we need to show that $\rho v \rho$ is square-free and contains only two occurrences of $\sigma$ and two occurrences of $\bar{\sigma}$. By 2., 3. and 4. of Lemma 10, any occurrence of $\sigma$ or $\bar{\sigma}$ is in $\rho$, so it only remains to show that $\rho v \rho$ is square-free.

Suppose, for the sake of contradiction, that there is a square in $\rho v \rho$, that is, there are words $x, y \in\{0,1,2\}^{*}$ and $u \in\{0,1,2\}^{+}$such that $\rho v \rho=x u u y$. Theorem 9 implies that $u$ cannot be a factor of $v$. One easily verifies that $\rho$ is square-free. Thus $x$ is a proper prefix of $\rho$ or $y$ is a proper suffix of $\rho$. Assume, without loss of generality, that $x$ is a proper prefix of $\rho$. Let $r$ be the nonempty suffix of $\rho$ such that $x r=\rho$. Fact 3. of Lemma 10 implies that the square $u u$ is not a factor of $\rho v_{1} v_{2} v_{3}$, thus $|u u| \geqslant\left|v_{1} v_{2} v_{3}\right|+2$ and $v_{1}$ is a factor of $u$. Thus $r v_{1}$ is a prefix of $u$. We have to distinguish between two different cases depending on the length of $r$.

Case $|\boldsymbol{r}| \geqslant 4$ : By hypothesis $|w| \geqslant 4$ and $\left|v_{2} \ldots v_{n}\right| \geqslant 5 \times 24>\left|v_{1}\right|+2|\rho| \geqslant\left|v_{1} r \rho\right|$ which gives $\left|r v_{1}\right|+\frac{|r v \rho|}{2}<|r v|$. We deduce that the $\left|r v_{1}\right|$ first letters of the second occurrence of $u$ do not overlap with the final occurrence of $\rho$. This implies that $r v_{1}$ is a factor of $v$. From Fact 1 . of Lemma 10, $v_{1}$ can only appear as the image of 0 , and thus $r$ must appear as the suffix of the image of a letter. However, it is easy to verify that $r$ is not the suffix of any image of a letter if $|r| \geqslant 4$ (it is enough to verify this with $|r|=4$ ), which is a contradiction.

\footnotetext{
${ }^{4}$ See the ancillary file verifying_lemma4.cpp accessible with the arXiv version at https://arxiv. org/abs/2102.00750.
} 
Case $|\boldsymbol{r}| \leqslant 3$ : Then $r$ is also the suffix of an image of 1 by $h$ (since 021 is suffix of any image of 1$)$. There is $v_{0} \in h(1)$ such that $u u$ is also a factor of $v_{0} v \rho$. By hypothesis, $w$ was chosen such that $10 w 01$ is square-free and Theorem 9 implies that there is no square in $v_{0} v$. Thus the square in $v_{0} v \rho$ overlaps with the final occurrence of $\rho$. By symmetry of the previous case, the square overlaps by at most 3 letters with the final occurrence of $\rho$ which implies that the square is also an image of $10 w 01$ which is a contradiction since any image of $10 w 01$ by $h$ is square-free by Theorem 9 .

We can deduce an exponential lower bound on the size of $\mathcal{N}_{n}$.

Theorem 12. For all $n \geqslant 7200$,

$$
\left|\mathcal{N}_{n}\right|>\frac{1.01^{n}}{18905}
$$

Proof. Let $P$ be the set of words over $\{0,1,2\}$ such that for any $w \in P,|w| \geqslant 4$ and $10 w 01$ is square-free. Lemma 11 implies that for all $n$

$$
\left|\mathcal{N}_{n}\right| \geqslant\left|\left\{v \in\{0,1,2\}^{n} \mid \exists w \in P, v \in h(0 w 0)\right\}\right| .
$$

The set of images by $h$ of two different words are disjoint, hence

$$
\left|\mathcal{N}_{n}\right| \geqslant\left|\left\{w \in P \mid h(0 w 0) \cap\{0,1,2\}^{n} \neq \emptyset\right\}\right| .
$$

Every letter has an image of length 24 and an image of length 25 by $h$. Thus for any integer $n \geqslant 12 \times 24 \times 25=7200$ and any word $u$ of length $\frac{n}{24}-12 \leqslant|u| \leqslant \frac{n}{24}$, $25|u| \geqslant n \geqslant 24|u|$ and $u$ admits at least an image of size $n$ by $h$. That is, for any $n \geqslant 7200$

$$
\left\{w \in P \mid h(0 w 0) \cap\{0,1,2\}^{n} \neq \emptyset\right\}|\geqslant|\left\{w \in P\left|\frac{n}{24}-12 \leqslant\right| 0 w 0 \mid \leqslant \frac{n}{24}\right\} \mid .
$$

By symmetry, there are exactly $\frac{C_{s q}(n)}{6}$ square-free words over $\{0,1,2\}$ of length $n$ starting by 10 .

A quick exhaustive search (a few minutes by hand) shows that every word avoiding 01 and starting with 0 has length at most 10. Since the longest square-free word over $\{1,2\}$ has length 3 , the longest square-free word avoiding 01 over $\{0,1,2\}$ has length at most 13. From any square-free $u$ word, we can obtain a square-free word ending with 01 by deleting a 01-free suffix of length at most 12 from the end of $u$. Since there are at most $2^{12}$ possible square-free suffixes to delete, there are at most $2^{12}$ such word $u$ that lead to the same word by this process. Thus for every integer $n$, there are at least $\frac{C_{s q}(n)}{6 \times 2^{12}}$ ternary square-free words of length between $n-12$ and $n$ starting with 10 and ending with 01 .

We can now apply Corollary 3,

$$
\left|\left\{w \in P \mid h(0 w 0) \cap\{0,1,2\}^{n} \neq \emptyset\right\}\right| \geqslant \frac{C_{s q}\left(\left\lfloor\frac{n}{24}\right\rfloor+2\right)}{24576}>\frac{1.3^{\left\lfloor\frac{n}{24}+2\right\rfloor}}{24576}>\frac{1.01^{n}}{18905} .
$$

Together with equation (3), we conclude $\left|\mathcal{N}_{n}\right|>\frac{1.01^{n}}{18905}$. 
We use the exponential lower bound to establish the existence of exponentially large $n$-good sets, but first we show one more property of nice words.

Lemma 13. Let $u, v \in \mathcal{N}$. If the word $\rho u \rho v \rho$ is not square-free then $u$ is a prefix of $v$ or $v$ is a suffix of $u$. In particular, if $|u|=|v|$ then $u=v$.

Proof. Let $u, v \in \mathcal{N}$ such that $u \neq v$. Let $w w$ be a square in $\rho u \rho v \rho$. Since $u$ and $v$ are nice, $\rho u \rho$ and $\rho v \rho$ are square-free. Hence the second occurrence of $\rho$ is a factor of $w w$. We also know that the only occurrences of $\sigma$ in $\rho u \rho v \rho$ (resp. of $\bar{\sigma}$ ) are the three occurrences inside each occurrence of $\rho$.

Suppose, for the sake of contradiction, that there are two non-empty words $u_{1}$ and $u_{2}$ such that $u=u_{1} u_{2}, w$ is a suffix of $\rho u_{1}$ and a prefix of $u_{2} \rho v \rho$. Since $w$ contains $\rho$ as a factors and that there are exactly three occurrences of $\rho$ this implies that $w=\rho u_{1}$ which is a contradiction with the fact that $u_{2} \rho v \rho$ does not starts with $\rho$. By symmetry, we reach a similar contradiction if we try to split $v$ in $v_{1} v_{2}$.

Hence there exists $\rho_{1}, \rho_{2}$ such that $\rho_{1} \rho_{2}=\rho=\bar{\sigma} 0 \sigma$ and $w$ is a suffix of $\rho u \rho_{1}$ and a prefix of $\rho_{2} v \rho$. Assume, without loss of generality, that $\sigma$ is a suffix of $\rho_{2}$ (otherwise $\bar{\sigma}$ is a prefix of $\rho_{1}$ and the rest of the argument is symmetric). Since the only occurrence of $\sigma$ in $\rho u \rho_{1}$ is inside $\rho$, we deduce that $\rho_{2} u \rho_{1}=w$. Since $w$ is a prefix of $\rho_{2} v \rho, u$ is a prefix of $v$ or $v$ is a prefix of $u$.

We can finally show the existence of exponentially large $n$-good sets.

Lemma 14. For any $n \geqslant 7200$, there exists an $n$-good set $S$ of size at least

$$
|S| \geqslant \frac{1.01^{n}}{40000\left(60 n^{2}+1\right)} .
$$

Proof. Let $\mathcal{N}_{n}^{\prime}$ be the set obtained by removing from $\mathcal{N}_{n}$ any prefix or suffix of every $l_{i}$ with $i \in\{2 n+100, \ldots, 7 n\}$ and by keeping for each pair of mirror images only the lexicographically smallest of the two. Each $l_{i}$ is responsible for removing at most two words from $\mathcal{N}_{n}$ so $\left|\mathcal{N}_{n}^{\prime}\right| \geqslant \frac{\left|\mathcal{N}_{n}\right|-10 n}{2} \geqslant \frac{1.01^{n}}{37810}-5 n$. Since $n \geqslant 7200$, we can simplify the bound

$$
\left|\mathcal{N}_{n}^{\prime}\right| \geqslant \frac{1.01^{n}}{40000}
$$

For any $u, v \in \mathcal{N}_{n}^{\prime}$, we say that $u$ forbids $v$ if $u \neq v$ and for some $i \in\{n+1, n+$ $2, \ldots, 5 n\}, \rho u \rho v \rho l_{i} \rho, \rho \bar{u} \rho v \rho l_{i} \rho, \rho u \rho \bar{v} \rho \bar{l}_{i} \rho$ or $\rho \bar{u} \rho \bar{v} \rho \bar{l}_{i} \rho$ contains a square.

We now count how many words $v$ are forbidden by a given $u$.

Let $u, v \in \mathcal{N}_{n}^{\prime}$ be such that $u \neq v$ and $\rho u \rho v \rho l_{i} \rho$ is not square-free. Lemma 13 implies that both $\rho u \rho v \rho$ and $\rho v \rho l_{i} \rho$ are square-free (since $u \neq v$ and $v$ is not a prefix of $l_{i}$ ). There is a non-empty suffix $u^{\prime}$ of $\rho u$ and a non-empty prefix $l^{\prime}$ of $l_{i} \rho$ such that the square $w w=u^{\prime} \rho v \rho l^{\prime}$. Moreover, there exist two non-empty words $v_{1}$ and $v_{2}$ such that $\sigma v \bar{\sigma}=v_{1} v_{2}$ and $w=u^{\prime} \bar{\sigma} 0 v_{1}=v_{2} 0 \sigma l^{\prime}$. Indeed, the middle of the square cannot be located outside of $\sigma v \bar{\sigma}$ since there would be too many occurences of $\sigma$ or $\bar{\sigma}$ on one side of the square. Finally, remark that either $v_{1}$ contains $\sigma$ as a prefix or $v_{2}$ contains $\bar{\sigma}$ as a suffix (both could be true). In both cases, using the fact that there are only two other occurrences of $\sigma$ and 
$\bar{\sigma}$, we deduce that $|w|=|v \rho|$. Thus $v_{1}$ is a prefix of $\sigma l_{i} \rho$ and $v_{2}$ is a suffix of $\rho u \bar{\sigma}$ and $v$ is uniquely determined by $u, l_{i}$ and the position of the square. There are $|v \rho|=n+39$ possible positions, less than $5 n$ possibles values for $l_{i}$, so $u$ forbids at most $(n+39) \times 5 n$ words because of $\rho u \rho v \rho l_{i}$. The count is similar for $\rho \bar{u} \rho v \rho l_{i}, \rho u \rho \bar{v} \rho \bar{l}_{i} \rho$ and $\rho \bar{u} \rho \bar{v} \rho \overline{l_{i}} \rho$, so $u$ forbids at most $(n+39) \times 5 n \times 4$ words. This is upper bounded by $30 n^{2}$ since $n>7200$.

Let $G$ be the graph whose vertices are the words from $\mathcal{N}_{n}^{\prime}$ and such that two words share an edge if one of them forbids the other one. The set of words corresponding to any independent set of $G$ is an $n$-good set. Let $S$ be the set of words corresponding to the largest independent set of $G$. Since every word forbids at most $30 n^{2}$ words, the average degree of the vertices of $G$ is at most $60 n^{2}$. By Corollary 5 , there is an independent set of size at least $\frac{\left|\mathcal{N}_{n}^{\prime}\right|}{60 n^{2}+1}$. Thus $|S| \geqslant \frac{1.01^{n}}{40000\left(60 n^{2}+1\right)}$.

\section{The final construction}

A graph $G^{\prime}$ is a $(\geqslant a, \leqslant b)$-subdivision of a graph $G$ if $G^{\prime}$ can be obtained by subdividing each edge of $G$ in at least $a$ and at most $b$ division vertices.

In Lemma 15, we use our results on $n$-goods sets to show that, if each edge of the graph is subdivided enough, but not too much, then we can nonrepetitively 3-color the resulting graph. To obtain Theorem 17, we then show that we can easily handle the edges that have too many subdivision vertices.

Lemma 15. Let $G$ be a graph and $n \geqslant 7200$ an integer such that $\pi^{\prime}(G) \leqslant \frac{1.01^{n}}{40000\left(60 n^{2}+1\right)}$. Then for any $(\geqslant 4 n+216, \leqslant 9 n)$-subdivision $G^{\prime}$ of $G$,

$$
\pi\left(G^{\prime}\right)=3
$$

Proof. Let $n, G$ and $G^{\prime}$ be as in the lemma statement. Let $C$ be a set of colors of size $\pi^{\prime}(G)$ and $\phi$ be a nonrepetitive edge $C$-coloring of $G$.

By Lemma 14, there is an $n$-good set $S$ such that $|S| \geqslant \pi^{\prime}(G)$. Let $f$ be an injective map from $C$ to $S$. Let $\vec{o}$ be an arbitrary orientation of the edges of $G$.

Let $\phi^{\prime}: V\left(G^{\prime}\right) \mapsto\{0,1,2\}$ be the 3-coloring of the vertices of $G^{\prime}$ such that

- every vertex of $G^{\prime}$ that corresponds to an original vertex of $G$ has color 0 ,

- for any edge $e$ from $G$ subdivided in $\left(v_{1}, \ldots, v_{d}\right)$ in $G^{\prime}$ with the $v_{i}$ ordered according to $\vec{o}(e)$, we let $t=d-116-2 n$ and the sequence of colors $\left(\phi^{\prime}\left(v_{1}\right), \ldots, \phi^{\prime}\left(v_{d}\right)\right)$ is equal to $\sigma f(\phi(e)) \rho l_{t} \rho f(\phi(e)) \bar{\sigma}$.

The equality $|\sigma f(\phi(e)) \rho|+|p f(\phi(e)) \bar{\sigma}|=2|\rho|+2|\sigma|+2 n=2 n+116$ implies

$$
\left|\sigma f(\phi(e)) \rho l_{t} \rho f(\phi(e)) \bar{\sigma}\right|=d .
$$

Thus $\phi^{\prime}$ is well-defined. Our goal is now to show that $\phi^{\prime}$ is nonrepetitive.

Since every edge of $G$ is subdivided at least $4 n+216$ times and at most $9 n$ times this implies that for each edge of $G$ subdivided into $d$ vertices, $2 n+100 \leqslant t \leqslant 7 n$. So the length of the $l_{i}$ allows us to apply Lemma 7 and Lemma 8. 
Let $\mathbf{p}$ be a path in $G^{\prime}$ whose two extremities do not belong to the subdivision of the same edge of $G$. Then it is a subpath of the subdivision of some path in $G$. Let $e_{1}, \ldots, e_{k}$ be this path of $G$. For all $i \in\{1, \ldots, k\}$, let $w_{i}=\phi\left(e_{i}\right)$, let $r_{i}$ be 0 if $\vec{o}\left(e_{i}\right)$ goes in the same direction as the orientation of the path and $r_{i}=1$ otherwise. For all $i \in\{1, \ldots, k\}$, let $d_{i}$ be the integer such that $e_{i}$ is subdivided into $d_{i}$ vertices in $G^{\prime}$ and let $t_{i}=d_{i}-116-2 n$. Then by definition the sequence of colors of the path $\mathbf{p}$ from $G^{\prime}$ is a factor of

$$
\rho \prod_{i=1}^{k} \overline{f\left(w_{i}\right) \rho l_{t_{i}} \rho f\left(w_{i}\right)^{r_{i}}} \rho .
$$

Moreover, since $\phi$ is nonrepetitive, $w_{1} \ldots w_{p}$ is square-free. By Lemma $7, \mathbf{p}$ is nonrepetitively colored by $\phi^{\prime}$.

Now we need to show that the same property holds if the two extremities of a path $\mathbf{p}$ of $G^{\prime}$ belong to the subdivision of the same edge. If the path is short and completely contained in an edge then this is in fact solved as the previous case. Then the remaining case is that $\mathbf{p}$ starts in the subdivision of an edge $e_{1}$ of $G$, then leaves this subdivision, and comes back to it by the other side. Let $e_{1}, e_{2}, \ldots, e_{n}, e_{1}$ be the edges of $G$ whose subdivision contains p. For all $i \in\{1, \ldots, k\}$, let $w_{i}=\phi\left(e_{i}\right)$. For all $i \in\{1, \ldots, k\}$, define $r_{i}$ and $t_{i}$ as in the previous case. Then there are two words $a$ and $b$ such that $\frac{f\left(w_{1}\right) \rho l_{t_{1}} \rho f\left(w_{1}\right)}{r_{1}} \rho=a b$ and such that the sequence of colors of $\mathbf{p}$ is a factor of

$$
b\left(\prod_{i=2}^{k}{\overline{f\left(w_{i}\right) \rho l_{t_{i}} \rho f\left(w_{i}\right)^{r}}}^{r_{i}} \rho\right) a .
$$

By Lemma 8, $\mathbf{p}$ is nonrepetitively colored by $\phi^{\prime}$.

We showed that every possible path of $G^{\prime}$ is nonrepetitively colored by $\phi^{\prime}$ which implies that $\phi^{\prime}$ is a nonrepetitive 3-coloring of $G^{\prime}$.

Lemma 16. Let $G$ be a graph and $H$ be a subdivision of $G$ then $\pi^{\prime}(H) \leqslant 2 \pi^{\prime}(G)+3$.

Proof. Let $\phi$ be a nonrepetitive edge coloring of $G$ over the set of colors $C$ of size $\pi^{\prime}(G)$. Let $C^{\prime}$ be the set of colors obtained by adding three new colors $\alpha, \beta, \gamma$ and for each color $c \in C$ a new color $c^{\prime}$.

Let $\phi^{\prime}$ be an edge coloring of $H$ such that for each edge $e$ of $G$ :

- if $e$ is not subdivided in $H$ then it has the same color in $H$ and in $G$,

- if $e$ is subdivided into two edges $e_{1}$ and $e_{2}$ then $\phi^{\prime}\left(e_{1}\right)=\phi(e)$ and $\phi^{\prime}\left(e_{2}\right)=\phi(e)^{\prime}$,

- if $e$ is subdivided in $k \geqslant 3$ edges $e_{1}, \ldots, e_{k}$, then $\phi^{\prime}\left(e_{1}\right)=\phi^{\prime}\left(e_{k}\right)=\phi(e)$ and the sequence $\phi^{\prime}\left(e_{2}\right) \ldots \phi^{\prime}\left(e_{k-1}\right)$ is a square-free word over $\{\alpha, \beta, \gamma\}$.

It is easy to verify that if there is a square in $\phi^{\prime}$ then the colors inherited from $\phi$ form a square on $G$. 
Theorem 17. Let $G$ be a graph and $c=\max \left\{29016,8 \frac{\log \left(2 \pi^{\prime}(G)+3\right)}{\log (1.01)}+216\right\}$. Then for any $(\geqslant c)$-subdivision $H$ of $G$,

$$
\pi(H)=3
$$

Proof. Let $n=\max \left\{7200,2 \frac{\log \left(2 \pi^{\prime}(G)+3\right)}{\log (1.01)}\right\}$. This implies $c=4 n+216$.

Let $G^{\prime}$ be a subdivision of $G$ such that $H$ is a $(\geqslant 4 n+216, \leqslant 9 n)$-subdivision of $G^{\prime}$. Let us first show that there exists such a graph $G^{\prime}$. For any integer $x>9 n$, let $\gamma(x)=\left\lfloor\frac{x}{4 n+217}\right\rfloor-1$. If an edge $e$ from $G$ needs to be subdivided with $x>9 n$ division vertices in $H$, then we subdivide $e$ with $\gamma(x)$ vertices in $G^{\prime}$ and we let $e_{1}, \ldots, e_{\gamma(x)+1}$ be the edges of $G^{\prime}$ corresponding to the subdivision of $G$. If for all $i \in\{1, \ldots, \gamma(x)\}$, we subdivide $e_{i}$ with $4 n+216$ vertices in $H$ and we subdivide $e_{\gamma(x)+1}$ with $x-\gamma(x)(4 n+216)-\gamma(x)$ vertices in $H$, then $e$ is subdivived with $\gamma(x)+\gamma(x)(4 n+216)+x-\gamma(x)(4 n+216)-\gamma(x)=$ $x$ subdivision vertices. One easily verifies that under the assumption that $n \geqslant 7200$, $4 n+216 \leqslant x-\gamma(x)(4 n+216)-\gamma(x) \leqslant 9 n$ so $H$ is a $(\geqslant 4 n+216, \leqslant 9 n)$-subdivision of $G^{\prime}$ as desired.

By Lemma $16,2 \pi^{\prime}(G)+3 \geqslant \pi^{\prime}\left(G^{\prime}\right)$. Let us now show that we can apply Lemma 15 to $G^{\prime}$ and $H$. We can verify by simple computation that $40000\left(60 n^{2}+1\right)<1.01^{n / 2}$ for any $n \geqslant 7200$ and

$$
\frac{1.01^{n}}{40000\left(60 n^{2}+1\right)} \geqslant 1.01^{n / 2} \geqslant 2 \pi^{\prime}(G)+3 \geqslant \pi^{\prime}\left(G^{\prime}\right) .
$$

So $n$ verifies the conditions of Lemma 15 . Since $H$ is a $(\geqslant 4 n+216, \leqslant 9 n)$-subdivision of $G^{\prime}$ we can apply Lemma 15 and we conclude that

$$
\pi(H)=3
$$

\section{Improving the coefficient and open questions}

We showed in Theorem 17 that there exists a function $f(n)=558 \log _{2}(n)+O(1)$ such that for any graph $G$ if there are at least $f\left(\pi^{\prime}(G)\right)$ division vertices per edge the resulting graph is nonrepetitively 3-colorable. This result is optimal in the sense that $\Omega(\log n)$ division vertices are needed on some edges of any nonrepetitively $O(1)$-colorable subdivision of $K_{n}[10]$. However, we can try to reduce the multiplicative constant 558. By being more careful on the computations, we can replace 558 by $\frac{4}{\log _{2}(\gamma(\mathcal{N}))}$ where $\gamma(\mathcal{N})$ is the growth rate of the set of nice words. By using our lower bound of 1.01 on the growth rate of the number of nice words (Theorem 12) we can take $f(n)=279 \log _{2}(n)+O(1)$. But we expect the growth rate to be much closer to 1.3 .

In fact, if instead of $\rho=\bar{\sigma} 0 \sigma$ we take any long enough square-free palindrome $\rho^{\prime}=\bar{\sigma}^{\prime} 0 \sigma^{\prime}$ then it is easy to adapt the proof from [14] to show that the growth rate of the set of square-free words that avoids $\sigma^{\prime}$ and $\bar{\sigma}^{\prime}$ can be arbitrarily close to 1.3. It probably does not change the growth rate to add the constraint that $\rho^{\prime} w \rho^{\prime}$ be square-free for every element $w$. However, we do not know how to prove this second point, but if it holds we can then replace 1.01 by 1.3 . This would allow to take $f(n)=10.56 \log _{2}(n)+O(1)$. We suspect that the coefficient 10.56 would still be far from optimal. 
Let us finally mention a few remaining open questions. We solved the first part of Wood's question but the second part remains open.

Question 18. Does every graph $G$ have a nonrepetitively 3-colourable subdivision with $O(\log \pi(G))$ division vertices per edge?

Our result improves the number of division vertices per edge over the result from Pezarski and Zmarz [12]. We say that a coloring is $\alpha$-free (resp. $\alpha^{+}$-free) if it does not contain any repetition of exponent at least $\alpha$ (resp. greater than $\alpha$ ). Hence, a coloring is nonrepetitive if it is 2-free. Ochem and Vaslet also strengthened [12] by showing that every graph can be subdivided into a graph that has a $\frac{7}{4}^{+}$-free 3 -coloring and that this is optimal [11]. It is natural to ask whether these two strengthenings can be combined.

Question 19. Does every graph $G$ have a subdivision $G^{\prime}$ with $\left.O(\log |V(G)|)\right)$ division vertices per edge such that $G^{\prime}$ has a $\frac{7}{4}^{+}$-free 3-coloring?

A similar question can be asked for the two other results of [11, Theorem 3.1].

Question 20. Does every graph $G$ have a subdivision $G^{\prime}$ with $\left.O(\log |V(G)|)\right)$ division vertices per edge such that $G^{\prime}$ has a $\frac{7}{3}^{+}$-free 2-coloring?

Does every graph $G$ have a subdivision $G^{\prime}$ with $\left.O(\log |V(G)|)\right)$ division vertices per edge such that $G^{\prime}$ has a $\frac{3}{2}^{+}$-free 4 -coloring?

\section{Acknowledgement}

I would like to thank the two anonymous referees for their helpful comments that improved the article.

\section{References}

[1] N. Alon, J. Grytczuk, M. Haluszcza, and O. Riordan. Nonrepetitive colorings of graphs. Random Structures \& Algorithms, 21:336-346, 2002.

[2] J. Barát and D. R. Wood. Notes on nonrepetitive graph colouring. Electronic Journal of Combinatorics, 15:\#R99, 2008.

[3] J. Currie, T. Harju, P. Ochem, N. Rampersad, Some further results on squarefree arithmetic progressions in infinite words, Theoretical Computer Science, 799:140-148, 2019.

[4] J. Berstel. Axel Thue's papers on repetitions in words: a translation. Publications du LaCIM 20, Université du Québec á Montréall, 1995.

[5] V. Dujmović, G. Joret, J. Kozik, and D. R. Wood. Nonrepetitive colouring via entropy compression. Combinatorica, 36(6):661-686, Dec 2016.

[6] P. Erdős and L. Lovász. Problems and results on 3-chromatic hypergraphs and some related questions. In Infinite and Finite Sets, vol. 10 of Colloq. Math. Soc. János Bolyai, pp. 609-627. North-Holland, 1975 
[7] D. Gonçalves, M. Montassier, and A. Pinlou. Entropy compression method applied to graph colorings. arXiv:1406.4380, 2014.

[8] J. Grytczuk. Nonrepetitive colorings of graphs-a survey. Int.J. Math. Math. Sci., 74639, 2007.

[9] J. Harant and S. Jendrol. Nonrepetitive vertex colorings of graphs. Discrete Mathematics, 312(2):374-380, 2012.

[10] J. Nešetřil, P. Ossona de Mendez, and D. R. Wood. Characterisations and examples of graph classes with bounded expansion. European Journal of Combinatorics, 33(3):350-373, 2011.

[11] P. Ochem and E. Vaslet. Repetition thresholds for subdivided graphs and trees. RAIRO-Theor. Inf. Appl., 46 (1) 123-130, 2012.

[12] A. Pezarski and M. Zmarz. Non-repetitive 3-coloring of subdivided graphs. Electronic Journal of Combinatorics, 16(1), \#N15 2009.

[13] M. Rosenfeld. Another approach to non-repetitive colorings of graphs of bounded degree. Electronic Journal of Combinatorics, 27(3), \#P3.43 2020.

[14] A. M. Shur. Two-Sided Bounds for the Growth Rates of Power-Free Languages. In: Diekert V., Nowotka D. (eds)Developments in Language Theory, DLT 2009. Lecture Notes in Computer Science, vol 5583.

[15] A. Thue. Über unendliche Zeichenreihen. 'Norske Vid. Selsk. Skr. I. Mat. Nat. Kl. Christiania, 7:1-22, 1906.

[16] A. Thue. Über die gegenseitige Lage gleicher Teile gewisser Zeichenreihen. Norske Vid. Selsk. Skr. I. Mat. Nat. Kl. Christiania,, 10:1-67, 1912.

[17] D. R. Wood. Nonrepetitive Graph Colouring. Electronic Journal of Combinatorics, \#DS24, 2021. 